\title{
A note on concave utility functions ${ }^{1}$
}

\author{
Martin Monti \\ Princeton University \\ Simon Grant \\ Rice University \\ Daniel Osherson \\ Princeton University
}

April 1, 2004 


\begin{abstract}
The classical theory of preference among monetary bets represents people as expected utility maximizers with nondecreasing concave utility functions. Critics of this account often rely on assumptions about preferences over wide ranges of total wealth. We derive a prediction of the theory that bears on bets at any fixed level of wealth, and test the prediction behaviorally. Our results are discrepant with the classical account. Competing theories are also examined in light of our data.
\end{abstract}

JEL classification: D81, C91.

keywords: gambling, risk aversion, concave utility function, expected utility, prospect theory 
An influential theory of preferences among bets represents people as expected utility maximizers with nondecreasing concave utility functions. In what follows, we shall call anyone who behaves this way a classical agent. The theory that people behave towards bets as if they were classical agents has been the subject of intense discussion, with alternative hypotheses prompted by experimental findings at variance with the classical account. ${ }^{1}$ A new kind of objection has recently been formulated by Rabin (2000a,b; Rabin \& Thaler, 2001). Let $(g, p, \ell)$ denote the bet yielding gain $\$ g$ with probability $p$ and loss $\$ \ell$ with probability $1-p$. Rabin deduces predictions of the form:

A classical agent who declines bet $(g, p, \ell)$ at wealth levels within interval $I$ will decline bet $\left(g^{\prime}, p^{\prime}, \ell^{\prime}\right)$ at wealth level $J$.

For example, he shows that:

(a) A classical agent who declines $(110, .5,100)$ at all wealth levels will decline $(X, .5,1000)$ for every $X$ and every wealth level.

(b) A classical agent who declines $(105, .5,100)$ through wealth level $\$ 350,000$ will decline $(635670, .5,4000)$ at wealth level $\$ 340,000$.

These predictions are all the more remarkable for being "parameter free." No assumptions about the utility curve are made except for its concavity throughout the domain of money. Rabin believes that the predictions do not conform to typical human preferences, hence most people are not classical agents. Indeed, Rabin \& Thaler (2001) conclude that the classical theory corresponds to the dead parrot in the famous sketch from Monty Python's Flying Circus, and they "aspire to have written one of the last articles debating the descriptive validity of the expected utility hypothesis" (p. 229).

Not everyone, however, acknowledges the infidelity of (a),(b) to human preferences. Watt (2002) and Palacios-Huerta, Serrano \& Volij (2002), for example, observe that the antecedent of (a) is difficult to verify empirically since it involves imagining choices under counterfactual circumstances of immense wealth. Prediction (b) is more trenchant in this regard, but it is not clear (pace the intuitions of Rabin and Thaler) what most people would do at the cited wealth levels. In particular, for someone as risk averse as indicated in the premise of (b), a $\$ 4,000$ loss might be a fearsome prospect when her fortune is limited to $\$ 340,000 .^{2}$

The present note attempts to preserve the spirit of Rabin's criticism while avoiding assumptions about behaviors across a wide range of wealth. We deduce a prediction about the choices of classical agents at a given level of wealth, and then present experimental evidence contrary to the prediction. Defects in the classical theory have 
been revealed in many experiments (e.g., the probability of choosing a given option can be increased by adding a new one; see Huber, Payne \& Puto, 1982; Simonson \& Tversky, 1992; Tentori, Osherson, Hasher \& May, 2001). The present demonstration is distinctive in its simplicity, and in its focus on the supposed concavity of the utility function.

\section{A simple constraint on the choices of classical agents}

As noted above, we use $(g, p, \ell)$ to denote the bet yielding gain $\$ g$ with probability $p$ and loss $\$ \ell$ with probability $1-p$. Let $\mathcal{A}$ be a classical agent with current wealth $w$ and (strictly increasing, concave) utility function $U$. Suppose that $\mathcal{A}$ is indifferent between accepting or rejecting the bet $\left(g, \frac{1}{2}, \ell\right)$, where $g \geq \ell>0$. Then $\frac{1}{2} U(w+g)+\frac{1}{2} U(w-\ell)=$ $U(w)$, so:

(1) $U(w+g)-U(w)=U(w)-U(w-\ell)$.

Directly from the concavity of $U$ :

$$
\begin{aligned}
& \frac{U(w)-U(w-g)}{g} \geq \frac{U(w)-U(w-\ell)}{\ell} \\
& \frac{U(w+g)-U(w)}{g} \leq \frac{U(w+\ell)-U(w)}{\ell}
\end{aligned}
$$

The latter two inequalities can be rewritten as:

$$
\begin{aligned}
& U(w)-U(w-g) \geq \frac{g}{\ell}[U(w)-U(w-\ell)] \\
& U(w+\ell)-U(w) \geq \frac{\ell}{g}[U(w+g)-U(w)]
\end{aligned}
$$

Substituting (1) into (2)a,b gives:

$$
\begin{aligned}
& U(w)-U(w-g) \geq \frac{g}{\ell}[U(w+g)-U(w)] \\
& U(w+\ell)-U(w) \geq \frac{\ell}{g}[U(w)-U(w-\ell)]
\end{aligned}
$$

Algebraic manipulation of (3)a,b yields:

$$
\frac{g}{\ell+g} U(w+g)+\frac{\ell}{\ell+g} U(w-g) \leq U(w)
$$




$$
\frac{g}{\ell+g} U(w+\ell)+\frac{\ell}{\ell+g} U(w-\ell) \geq U(w)
$$

Thus, $\mathcal{A}$ will decline $(g, p, g)$ for any $p<g /(\ell+g)$, and will accept $(\ell, p, \ell)$ for any $p>g /(\ell+g)$. Summarizing:

(5) Any classical agent who is indifferent between accepting or rejecting $\left(g, \frac{1}{2}, \ell\right)$ will decline $(g, p, g)$ for any $p<g /(\ell+g)$ and accept $(\ell, p, \ell)$ for any $p>g /(\ell+g)$.

Note that (5) does not depend on $\mathcal{A}$ 's level of wealth. Nor does (5) depend on the particular shape of $\mathcal{A}$ 's utility function, beyond concavity and monotonicity. It is also worth observing that for $p$ near $g /(\ell+g)$ (with $g>\ell$ ), both $(g, p, g)$ and $(\ell, p, \ell$ ) have positive expected monetary value $(\mathrm{EMV})$, with the first bet more favorable than the second. So the bounds in (5) express the fact that a classical agent may accept a bet whose EMV is less than a rejected bet in order to avoid exposure to risk.

\section{Experiment}

If people respond to bets like classical agents then (5) yields clear predictions about their preferences. To test the predictions, we asked college students to create bets to which they were indifferent, and to evaluate other bets. These tasks were carried out in four successive stages.

Stage 1. The students were first asked to adjust the value of the gains $X, Y, Z$ in bets $(X, .5,10),(Y, .5,200)$, and $(Z, .5,1100)$ to the smallest amounts that render each bet barely acceptable. The bets were presented in the order just indicated. Although it was made clear that all bets in the study were hypothetical, the students were urged to imagine them as genuine options available immediately. It was emphasized that each bet was to be considered individually (not conditional on having accepted other bets presented earlier). The numbers issuing from this part of the procedure are denoted by $G_{10}, G_{200}, G_{1100}$. We interpret each subject to be close to indifferent about $\left(G_{10}, .5,10\right)$, $\left(G_{200}, .5,200\right)$, and $\left(G_{1100}, .5,1100\right)$. From (5) we therefore obtain:

(6) If a given subject is a classical agent then s/he will accept $(10, p, 10)$ if

$$
p>\frac{G_{10}}{10+G_{10}}
$$

and similarly for $(200, p, 200)$ and $(1100, p, 1100)$. 
Stage 2. Next, each participant adjusted the losses $X, Y, Z$ in bets $(10, .5, X)$, $(200, .5, Y)$, and $(1100, .5, Z)$ to the largest amounts that render each bet barely acceptable (bets presented in the order indicated). The numbers issuing from this part of the procedure are denoted by $L_{10}, L_{200}, L_{1100}$. We interpret each subject to be close to indifferent about $\left(10, .5, L_{10}\right),\left(200, .5, L_{200}\right)$, and $\left(1100, .5, L_{1100}\right)$. From (5) we therefore obtain:

(7) If a given subject is a classical agent then s/he will decline $(10, p, 10)$ if

$$
p<\frac{10}{L_{10}+10}
$$

and similarly for $(200, p, 200)$ and $(1100, p, 1100)$.

Stage 3. Participants were then asked whether they would accept each bet in a series of twelve. The twelve bets were presented in random order, and a yes/no decision was made to each in turn. Six of the bets were "fillers," designed to mask the focus of the experiment. The other six had the following forms.

\begin{tabular}{|lll} 
a) & $(10, p, 10)$ & where $p=.95 \times\left(10 /\left(L_{10}+10\right)\right)$ \\
b) $\quad(200, p, 200)$ & where $p=.95 \times\left(200 /\left(L_{200}+200\right)\right)$ \\
c) $\quad(1100, p, 1100)$ & where $p=.95 \times\left(1100 /\left(L_{1100}+1100\right)\right)$ \\
d) $\quad(10, p, 10)$ & where $p=1.05 \times\left(G_{10} /\left(G_{10}+10\right)\right)$ \\
e) $\quad(200, p, 200)$ & where $p=1.05 \times\left(G_{200} /\left(G_{200}+200\right)\right)$ \\
f) & $(1100, p, 1100)$ & where $p=1.05 \times\left(G_{1100} /\left(G_{1100}+1100\right)\right)$
\end{tabular}

Thus, if our participants were classical agents, (6) and (7) predict that they decline bets (8)a,b,c and accept bets (8)d,e,f. Note our use of probabilities that fall decisively on the active side of each boundary (either $95 \%$ of the highest unacceptable probability or $105 \%$ of the least acceptable one).

Stage 4. Finally, for each of three bets of form $(x, p, x)$, participants were asked to specify the minimum probability $p$ that renders $(x, p, x)$ barely acceptable. The three bets were $(10, p, 10),(200, p, 200),(1100, p, 1100)$, evaluated in that order. According to (6) and (7), the value of $p$ chosen for the three bets should lie in the following intervals. 


$$
\begin{aligned}
& p \text {-interval for }(10, p, 10)=\left[\frac{10}{L_{10}+10}, \frac{G_{10}}{10+G_{10}}\right] \\
& p \text {-interval for }(200, p, 200)=\left[\frac{200}{L_{200}+200}, \frac{G_{200}}{200+G_{200}}\right] \\
& p \text {-interval for }(1100, p, 1100)=\left[\frac{1100}{L_{1100}+1100}, \frac{G_{1100}}{1100+G_{1100}}\right]
\end{aligned}
$$

Method. As subjects, we recruited 23 undergraduates at Rice University, and 28 undergraduates at Princeton University ( $52 \%$ female). All fulfilled course requirements through their participation. The students first received instruction from an experimenter, then worked individually at computer consoles where instructions were repeated in text format. The experiment required about 20 minutes to complete. There were no appreciable differences between Rice and Princeton students, so all participants are grouped in the analyses that follow. Note that the predictions issuing from (5) of the Classical Theory may be tested entirely on a within subject basis.

\section{Results}

\section{- INSERT TABle 1 ABOUT HeRE - -}

Table 1 shows the median dollar amounts chosen in Stages 1 and 2. Although the medians seem reasonable, analysis at the within-subject level reveals conflict with the Classical Theory. We illustrate with one student who set $G_{10}=11$ and $L_{10}=6$ in Stages 1 and 2 of the experiment. If he is a classical agent, (6) and (7) imply that he will accept $(10, p, 10)$ if $p>\frac{11}{21}$ and reject the same bet if $p<\frac{10}{16}$. Hence, indifference to $(10, p, 10)$ requires $p \in\left[\frac{10}{16}, \frac{11}{21}\right]$, which is impossible. Call such a subject (for whom $\left.\frac{10}{L_{10}+10}>\frac{G_{10}}{10+G_{10}}\right)$ incoherent at level 10, and similarly for levels 200 and 1100 . The middle column of Table 2 shows that incoherence was frequent at each level. Indeed, 46 of the 51 students were incoherent at some level.

\section{- INSERT TABLE 2 ABOUT HERE --}

The degree of (in)coherence at a given level is measured by the difference between the upper and lower bounds exhibited in (9). Negative values are inconsistent with the classical theory. Table 2 shows incoherence at all three levels according to this measure. At level 1100, the average distance from the lower to the upper bound in (9) is -.023, significantly less than $0.0(t=-2.37)$. Thus, at level 1100 , the computed upper bound is reliably below the computed lower bound. 
Another sense in which the students did not behave like classical agents emerges from their attitudes to the bets in (8). According to (6) and (7), the students should decline bets (8)a,b,c and accept bets (8)d,e,f. The number of times these predictions were violated is recorded in Table 3. Each of the predictions (8)a,b was violated by a majority of the students. In the first of these cases, the majority is significantly greater than expected from uniform random choice $($ prob $<.001$ by a binomial test with parameter .5). Of the 51 students, 44 violated at least one of the predictions (8)a,b,c, and 30 violated at least one of (8)d,e,f.

\section{- INSERT TABLE 4 ABOUT HERE -}

The probabilities specified in Stage 4 of the Experiment (henceforth called " $p$ values") are shown in the first two columns of Table 4. Starred results occurred significantly more often than predicted by a coin-flip model (prob $<.001$ by binomial test). The increase in probability across the three levels is reliable by correlated $t$-test $($ prob <.001), and appears to reflect aversion to risk. The table also shows that many of the $p$-values fell outside of the intervals displayed in (9). Indeed, at each level, a reliable majority of the subjects failed to honor at least one boundary of the interval. (It is possible to honor neither boundary in case of incoherent bounds.)

\section{- INSERT TABLE 5 ABOUT HERE —-}

To quantify the discrepancy between chosen $p$-values and their predicted intervals in $(9)$, let $p_{10}$ be the probability that a given subject chose in Stage 4 , and similarly for $p_{200}$ and $p_{1100}$. Define the undershoot for level 10 to be:

$$
0 \text { if } p_{10} \geq \frac{10}{L_{10}+10}, \text { and otherwise } \frac{10}{L_{10}+10}-p_{10}
$$

Thus, the undershoot for level 10 is the distance (if any) from $p_{10}$ upward to the lower bound specified in (9). Define the overshoot for level 10 to be:

$$
0 \text { if } p_{10} \leq \frac{G_{10}}{10+G_{10}} \text {, and otherwise } p_{10}-\frac{G_{10}}{10+G_{10}}
$$

The overshoot for level 10 is thus the distance (if any) from $p_{10}$ downwards to the upper bound specified in (9). Let the undershoot and overshoot for levels 200 and 
1100 be defined similarly. Table 5 shows the undershoots and overshoots at each level. Thus, the average distance from $p_{10}$ upward to the lower bound for $(10, p, 10)$ shown in (9) is .076 (S.D. $=.099$ ). (If $p_{10}$ for a given subject is above the bound then his/her contribution to the mean is zero.) The average distance from $p_{10}$ downward to the upper bound for $(10, p, 10)$ shown in $(9)$ is .027 (S.D. $=.050)$. (If $p_{10}$ for a given subject is below the bound then his/her contribution to the mean is zero.) The other numbers in Table 5 are interpreted similarly. The table shows that the undershoots were greater for level 10 compared to 200, and greater for 200 compared to 1100; likewise, the overshoots were greater for level 1100 compared to 200, and for 200 compared to 10 . All the means differ reliably from each other $($ prob $<.02)$ by correlated $t$-test except for the undershoots at levels 200 and $1100(t=1.78)$, and the undershoot versus overshoot at level $200(t=1.69)$. It is thus clear that the participants deviated from classical agents in a systematic rather than random way.

Call a subject classical at level 10 if her $p$-value for that level lies in the (coherent) $p$-interval $\left[\frac{10}{L_{10}+10}, \frac{G_{10}}{10+G_{10}}\right]$, and similarly for levels 200 and 1100 . Only 7 subjects were classical at level 10, 4 at level 200, and 1 at level 1100. Not a single subject behaved like a classical agent at all three levels.

\section{Alternatives to the classical theory}

Consider again an agent $\mathcal{A}$ whose preferences among bets are governed by nondecreasing utility curve $U$. Suppose that $\mathcal{A}$ is indifferent between accepting and rejecting the bet $(g, .5, \ell)$, where $g \geq \ell>0$. Then $\frac{1}{2} U(w+g)+\frac{1}{2} U(w-\ell)=U(w)$, and once again we obtain equality $(1)$, repeated here:

(1) $U(w+g)-U(w)=U(w)-U(w-\ell)$.

If $U$ is concave (respectively, convex) in the domain of gains then:

$$
\frac{U(w+g)-U(w)}{g} \leq(\text { respectively, } \geq) \frac{U(w+\ell)-U(w)}{\ell}
$$

These inequalities concern the "domain of gains" because only increases to $w$ are at issue. Similarly, if $U$ is concave (respectively, convex) in the domain of losses then:

$$
\frac{U(w)-U(w-g)}{g} \geq(\text { respectively, } \leq) \frac{U(w)-U(w-\ell)}{\ell}
$$


Substituting (1) into the latter inequalities produces:

$$
\begin{aligned}
& U(w+\ell)-U(w) \geq(\text { respectively, } \leq) \frac{\ell}{g}[U(w)-U(w-\ell)] \\
& U(w)-U(w-g) \geq(\text { respectively, } \leq) \frac{g}{\ell}[U(w+g)-U(w)]
\end{aligned}
$$

Algebraic manipulation then yields the following.

(a) If $U$ is concave (respectively, convex) in the domain of gains then:

$$
\frac{g}{\ell+g} U(w+\ell)+\frac{\ell}{\ell+g} U(w-\ell) \geq(\text { respectively, } \leq) U(w)
$$

(b) If $U$ is concave (respectively, convex) in the domain of losses then:

$$
\frac{g}{\ell+g} U(w+g)+\frac{\ell}{\ell+g} U(w-g) \leq(\text { respectively, } \geq) U(w)
$$

If $U$ is concave in both the domain of gains and the domain of losses then we recover our classical agent, described by (5). If $U$ is concave in the domain of gains and convex in the domain of losses then $\mathcal{A}$ resembles the kind of agent depicted in Prospect Theory (Kahneman \& Tversky, 1978). In this case, (10) implies that $\mathcal{A}$ will accept both $(g, p, g)$ and $(\ell, p, \ell)$ if $p>g /(\ell+g)$. [Hence, the probability that $\mathcal{A}$ assigns in Stage 4 of the experiment must lie below $X /\left(L_{X}+X\right)$ and $G_{X} /\left(X+G_{X}\right)$, for each level $X \in\{10,200,1100\}$.] In contrast, if $U$ is convex in the domain of gains and concave in the domain of losses then $\mathcal{A}$ is more like the agent discussed by Friedman \& Savage (1948). In this case, (10) implies that $\mathcal{A}$ will decline both $(g, p, g)$ and $(\ell, p, \ell)$ if $p<g /(\ell+g)$. [Hence, the probability that $\mathcal{A}$ assigns in Stage 4 of the experiment must lie above $X /\left(L_{X}+X\right)$ and $G_{X} /\left(X+G_{X}\right)$, for each level $X \in\{10,200,1100\}$.] Let us introduce the following terminology.

(11) Definition: Let $X \in\{10,200,1100\}$ be given. Let $G_{X}$ and $L_{X}$ be the values assigned in Stages 1 and 2 of the experiment, and let $p_{X}$ be the probability assigned in Stage 4.

(a) A subject is $K T$ at level $X$ if and only if $p_{X}$ bounded above by both $X /\left(L_{X}+X\right)$ and $G_{X} /\left(X+G_{X}\right)$. (KT abbreviates "Kahneman \& Tversky".)

(b) A subject is $F S$ at level $X$ if and only if $p_{X}$ is bounded below by both $X /\left(L_{X}+X\right)$ and $G_{X} /\left(X+G_{X}\right)$. (FS abbreviates "Friedman \& Savage".) 
The definition provides apt characterizations of Kahneman \& Tversky (1978) and Friedman \& Savage (1948) only if

$$
G_{X} \geq X \geq L_{X} \text { and } p_{X} \geq .5
$$

inasmuch as these inequalities were assumed for the developments above. In what follows, at each level $X$ we therefore exclude subjects who violated (12).

Consider a subject $S$ who satisfies (12). The weak inequalities appearing in Definition (11) allow $S$ to be more than one of KT, FS, and classical. It is also possible for $S$ to be none of the three types. For example, one subject (mentioned at the beginning of the Results section) chose $G_{10}=11, L_{10}=6, p_{10}=.55$; calculation of $10 /\left(L_{10}+10\right)$ and $G_{10} /\left(X+G_{10}\right)$ reveals that .55 is neither above both these bounds (thus ruling out FS), nor below both (ruling out KT), nor "in between" (since the bounds are inverted, which rules out classical).

\section{- Insert TABle 6 ABOUt HeRE -}

Table 6 shows the number of subjects of each kind (KT, FS, classical) at the three levels. It also exhibits the number of subjects (out of 51) conforming to (12). At each level, a large majority is either KT or FS; few are classical. The difference in proportions of KT and classical subjects is reliable at each level ( prob $<.01)$; the same is true of the differences between FS and classical subjects. There were reliably more KT than FS subjects at level 10 ( prob <.02), and the reverse at level 1100 ( prob <.01); there is no reliable difference at level 200.

A different alternative to the classical theory posits "first-order" aversion to risk, that is, the disinclination to accept fair gambles even with tiny stakes; in contrast, classical agents are indifferent to them; see Segal \& Spivak (1990). In an influential article, Gul (1991) offers a generalization of utility theory that is consistent with firstorder aversion. It implies that an agent with wealth $w$ and utility function $U$ will accept $(g, p, \ell)$ if and only if

$$
U(w)<U_{w}(g, p, \ell)=\frac{p}{p+(1-p) \lambda} U(w+g)+\frac{(1-p) \lambda}{p+(1-p) \lambda} U(w-\ell),
$$

where $\lambda$ is a parameter characterizing the agent's aversion to disappointment; the standard theory is recovered at $\lambda=1$. It follows easily that for any level $w$ of wealth, the marginal change in $U_{w}(\epsilon, .5, \epsilon)$ goes to $\frac{1-\lambda}{1+\lambda} U^{\prime}(w)$ as $\epsilon \rightarrow 0$, signifying first-order aversion to loss when $\lambda>1$. 
If Gul's model is descriptively accurate with concave $U$, it might be taken as partial vindication of the classical theory. To investigate this possibility, call an agent Gul if her preferences for gambles are governed by inequality (13), where $U$ is concave and $\lambda>0$. By an argument similar to the one advanced earlier, we can show:

Any Gul agent who is indifferent between accepting or rejecting $\left(g, \frac{1}{2}, \ell\right)$ will decline $(g, p, g)$ for any $p<g /(\ell+g)$ and accept $(\ell, p, \ell)$ for any $p>g /(\ell+g)$.

Since this is the same prediction as (5) for classical agents, our experimental results conflict with (13) as much as they conflict with the classical theory. It therefore appears that adding first-order risk-aversion to the concavity assumption may not be sufficient to describe real choices among lotteries.

\section{Discussion}

The experimental results are discrepant with the hypothesis that college students behave like classical agents when evaluating bets. Instead of behaving classically, Table 6 suggests that at low stakes, most subjects choose as if their utility for money were concave for gains and convex for losses (as suggested by Kahneman \& Tversky, 1978); the reverse patterns holds for high stakes (conforming to Friedman \& Savage, 1948). The discrepancy with the classical theory thus appears to be systematic. ${ }^{3}$

Two caveats must be entered. First, the bets in our study were hypothetical so it remains possible that the students would respond like classical agents if faced with the real thing. ${ }^{4}$ Second, people might resemble classical agents better when they are led to conceptualize bets in terms of overall wealth, e.g., in terms of $U(w-\$ 10)$ rather than a decontextualized $\$ 10$ loss. It is well known that attitudes towards gains versus losses are asymmetric in ways that apply less to overall wealth. ${ }^{5}$

The results nonetheless suggest that the classical theory of risk aversion is qualitatively inaccurate. For, choices deviate in systematic fashion from predictions that are independent of parametric assumptions about the utility curve, beyond concavity itself. In this sense, our findings sustain the principal thesis advanced in Rabin (2000a,b). 


\section{Notes}

${ }^{1}$ See Kahneman \& Tversky (2000) for assessment of the descriptive realism of utility theory, and alternative models. For a history of the classical theory, see Arrow (1971); its success in behavioral prediction is reviewed in Camerer (1995).

${ }^{2}$ LeRoy (2003) offers reason to doubt that most people reject small, unfavorable gambles like $(105, .5,100)$. But see Rabin \& Thaler's (2002) response to critics, and the data they cite about risk aversion in gambles with small stakes.

${ }^{3}$ Since gambles were always evaluated in order of increasing stakes - 10 to 200 to 1100 - the apparent interaction between stakes and conformity to KT versus FS may be partly an order effect.

${ }^{4}$ The impact of real stakes on conformity to economic postulates, however, is not straightforward. See Camerer \& Hogarth (1999) for a review of findings.

${ }^{5}$ See Tversky \& Bar-Hillel (1983), Kahneman, Knetsch \& Thaler (1991), Kahneman \& Tversky (1995), and references cited there. 


\section{References}

Arrow, Kenneth J. (1971) Essays in the Theory of Risk Bearing (Chicago IL: Markham Publishing Company)

Camerer, Colin (1995) 'Individual Decision Making.' In Handbook of Experimental Economics, ed. John Kagel and Al Roth (Princeton NJ: Princeton University Press)

Camerer, Colin, and Robin Hogarth (1999) 'The effects of financial incentives in economic experiments: A review and capital-labor-production framework.' Journal of Risk and Uncertainty 18, 7-42

Friedman, Milton, and Leonard Savage (1948) 'The utility analysis of choices involving risk.' Journal of Political Economy 56(4), 279-304

Gul, Faruk (1991) 'A theory of disappointment aversion.' Econometrica 59(3), 667-686

Huber, Joel, John W. Payne, and Chris Puto (1982) 'Adding asymmetrically dominated alternatives: violations of regularity and the similarity hypothesis.' Journal of Consumer Research 9, 90-98

Kahneman, Daniel, and Amos Tversky (1979) 'Prospect Theory: An Analysis of Decision under Risk.' Econometrica 47(2), 263-91

- (1995) 'Conflict Resolution: A Cognitive Perspective.' In Barriers to Conflict Resolution, ed. Kenneth J. Arrow (New York NY: W.W. Norton)

Kahneman, Daniel, and Amos Tversky, eds (2000) Choices, Values, and Frames (Cambridge, England: Cambridge University Press)

Kahneman, Daniel, John L. Knetsch, and Richard Thaler (1991) 'Anomalies: The Endowment Effect, Loss Aversion, and Status Quo Bias.' Journal of Economic Perspectives 5(1), 193-206

LeRoy, Stephen (2003) 'Expected utility: a defense.' Economics Bulletin 7(7), 1-3

Palacios-Huerta, Ignacio, Robert Serrano, and Oscar Volij (2002) 'Rejecting Small Gambles Under Epected Utility.' Manuscript

Pratt, John (1964) 'Risk Aversion in the Small and in the Large.' Econometrica $32,122-136$

Rabin, M. (2000a) 'Risk Aversion and Expected-Utility: A Calibration Theorem.' Econometrica 68, 1281-1292

Rabin, M., and R. Thaler (2001) 'Risk aversion.' Journal of Economic Perspectives 15, 219-232

Rabin, Matthew (2000b) 'Diminishing Marginal Utility of Wealth Cannot Explain Risk Aversion.' In Choices, Values, and Frames, ed. Daniel Kahneman and Amos Tversky (Cambridge, England: Cambridge University Press)

Rabin, Matthew, and Richard Thaler (2002) 'Response.' Journal of Economic 
Perspectives 16(2), 229-230

Segal, Uzi, and Avia Spivak (1990) 'First-order versus second-order risk aversion.' Journal of Economic Theory 51, 111-125

Simonson, Israel, and Amos Tversky (1992) 'Choice in context: Tradeoff contrast and extremeness aversion.' Journal of Marketing Research 29, 281-287

Tentori, Katya, Daniel Osherson, Lynn Hasher, and Cynthia May (2001) 'Wisdom and aging: Irrational preferences in college students but not older adults.' Cognition 81/3, B87-B96

Tversky, Amos, and Maya Bar-Hillel (1983) 'Risk: The Long and the Short.' Journal of Experimental Psychology: Learning, Memory and Cognition 9(4), 713-117

Watt, Richard (2002) 'Defending expected utility theory.' Journal of Economic Perspectives 16(2), 227-230 
TABLE 1

$\underline{\text { Median gains and losses chosen in Stages } 1 \text { and } 2(N=51)}$

\begin{tabular}{|c|c||c|c|}
\hline $\begin{array}{c}\text { question } \\
\text { format }\end{array}$ & $\begin{array}{c}\text { median } \\
\text { chosen } \\
\text { gain } X\end{array}$ & $\begin{array}{c}\text { question } \\
\text { format }\end{array}$ & $\begin{array}{c}\text { median } \\
\text { chosen } \\
\text { loss } X\end{array}$ \\
\hline$(X, .5,10)$ & 20 & $(10, .5, X)$ & 5 \\
$(X, .5,200)$ & 500 & $(200, .5, X)$ & 75 \\
$(X, .5,1100)$ & 3500 & $(1100, .5, X)$ & 255 \\
\hline
\end{tabular}


TABLE 2

$\underline{\text { Number incoherent at each level, and mean width of coherent interval }(N=51)}$

\begin{tabular}{|c|c|c|}
\hline Level & $\begin{array}{c}\text { Number } \\
\text { incoherent }\end{array}$ & Mean (S.D.) \\
\hline 10 & 22 & $-.005(.084)$ \\
200 & 31 & $-.031(.017)$ \\
1100 & 35 & $-.023^{*}(.010)$ \\
$\begin{array}{c}\text { at least } \\
\text { one level }\end{array}$ & 46 & \\
\hline
\end{tabular}

*At level 1100, the average distance from the lower to the upper bound in (9) is significantly less than $0.0(t=-2.37)$. 


\section{TABLE 3}

$\underline{\text { Number of Stage } 3 \text { choices inconsistent with the classical theory }(N=51)}$

\begin{tabular}{|c|c|c|c|}
\hline & \multicolumn{3}{|c|}{ Levels } \\
\hline prediction & 10 & 200 & 1100 \\
\hline (8)a,b,c & $37^{*}$ & 31 & 20 \\
(8)d,e,f & 6 & 13 & 19 \\
\hline
\end{tabular}

Note. Inconsistency with (8)a,b,c requires accepting a gamble predicted to be rejected. Inconsistency with $(8) \mathrm{d}, \mathrm{e}, \mathrm{f}$ is the reverse. The starred result occurred significantly more often than predicted by a coin-flip model $($ prob $<.001$ by binomial test). 
TABLE 4

$\underline{\text { Probabilities produced in Stage } 4(N=51)}$

\begin{tabular}{|c|c|c|c|c|}
\hline Bet & $\begin{array}{c}\text { Mean } \\
\text { p-value } \\
\text { (and S.D.) }\end{array}$ & $\begin{array}{c}\text { Number } \\
\text { below } \\
\text { lower } \\
\text { bound }\end{array}$ & $\begin{array}{c}\text { Number } \\
\text { above } \\
\text { upper } \\
\text { bound }\end{array}$ & $\begin{array}{c}\text { Number } \\
\text { outside } \\
\text { interval }\end{array}$ \\
\hline$(10, p, 10)$ & $.585(.146)$ & 31 & 18 & $44^{*}$ \\
$(200, p, 200)$ & $.738(.120)$ & 23 & 31 & $47^{*}$ \\
$(1100, p, 1100)$ & $.843(.107)$ & 15 & $40^{*}$ & $50^{*}$ \\
\hline
\end{tabular}

* Starred results occurred significantly more often than predicted by a coin-flip model $($ prob $<.001$ by binomial test $)$. 


\section{TABLE 5}

$\underline{\text { Undershoot and overshoot in Stage } 4(N=51)}$

\begin{tabular}{|c||c|c|}
\hline Bet & $\begin{array}{c}\text { Mean } \\
\text { undershoot } \\
\text { (and S.D.) }\end{array}$ & $\begin{array}{c}\text { Mean } \\
\text { overshoot } \\
\text { (and S.D.) }\end{array}$ \\
\hline$(10, p, 10)$ & $.076(.099)$ & $.027(.050)$ \\
$(200, p, 200)$ & $.032(.049)$ & $.061(.099)$ \\
$(1100, p, 1100)$ & $.011(.029)$ & $.093(.088)$ \\
\hline
\end{tabular}




\section{TABLE 6}

Number of Stage 4 probabilities within intervals predicted by different theories

\begin{tabular}{|c|c|c|c|}
\hline & \multicolumn{3}{|c|}{ Level: } \\
\hline & $\begin{array}{c}10 \\
(N=46)\end{array}$ & $\begin{array}{c}200 \\
(N=48)\end{array}$ & $\begin{array}{c}1100 \\
(N=50)\end{array}$ \\
\hline Theory: & & & \\
\hline $\mathrm{KT}$ & 27 & 17 & 10 \\
\hline FS & 16 & 24 & 34 \\
\hline Classical & 6 & 3 & 1 \\
\hline
\end{tabular}

Note. At each level $X, N$ is the number of subjects (out of 51) who satisfied the inequalities (12). 